\title{
Custos morais e Direito Comparado ${ }^{1}$ Moral costs and Comparative Law
}

\section{Giuseppe Bellantuono 2}

Resumo: Para Guido Calabresi, os custos morais surgem quando a comunidade rejeita estruturas autoritárias ou de mercado que estabelecem preços para bens meritórios, ou recusa que o acesso a esses bens dependa da riqueza individual. Reconhecer que aos custos morais deve ser atribuída a mesma importância dos custos de transação significa enfrentar duas dificuldades metodológicas: primeira, a identificação dos contextos em que os custos morais são relevantes; segunda, a seleção das estruturas autoritárias ou de mercado capazes de geri-los. Neste artigo, propõe-se uma perspectiva comparativa para enfrentar ambas as dificuldades. Mais especificamente, é proposta uma análise em três estágios para identificar os contextos nos quais os custos morais são relevantes: a seleção de áreas nas quais é plausível esperar que os custos

1 Traduzido por Marcos Henrique Costa Leroy, Doutorando e Mestre em Propriedade Intelectual na área de estudo de Direito Econômico e Desenvolvimento pela Universidade Federal de Minas Gerais. Título original do artigo: "Costi Morali e Diritto Comparato".

2 Professor de Direito Privado Comparado da Università degli Studi di Trento. Agradeço a Massimiliano Vatiero pelos comentários. Erros e omissões são meus. 
morais e os custos de transação se movam em direções opostas (primeira fase); a avaliação das estruturas institucionais que em cada sistema jurídico contribuem para reduzir ou aumentar as desigualdades (segunda fase); e o uso de dados empíricos sobre a difusão de certos princípios morais em diferentes países para confirmar a relevância dos custos morais em contextos específicos (terceira fase). Com referência à gestão dos custos morais, são listados alguns fatores que devem ser levados em consideração para a seleção de estruturas autoritárias ou de mercado. Além disso, sugere-se que a análise comparativa deve servir para avaliar quais dessas estruturas são compatíveis com cada contexto institucional.

Palavras-chave: Direito Comparado; Custos Morais; Calabresi; Direito e Economia.

Abstract: According to Calabresi, moral costs arise when people reject authoritarian or market structures imposing prices on merit goods, or reject trading of those goods being dependent on income. Acknowledging the relevance of moral costs, and placing them on the same plane as traditional transaction costs, requires to address two methodological challenges: first, how to identify the contexts in which moral costs are relevant; second, how to select the best authoritarian/market structures to govern them. In this paper, it is argued that a comparative perspective helps address both challenges. More specifically, it is proposed a threepronged analysis to identify the contexts in which moral costs are relevant: selecting those areas where it can be expected that moral costs and transaction costs move in opposite directions (first prong); assessing to what extent each legal system under scrutiny includes institutional structures more or less favourable to 
curbing inequalities (second prong); using crosscountry empirical data on moral values to confirm the relevance of moral costs in specific contexts (third prong). With regard to the governance of moral costs, I list several factors which should be taken into account when designing authoritarian or market structures. I also argue that the role of comparative analysis should be to evaluate which structure fits each institutional context.

Keywords: Comparative Law; Moral Costs; Calabresi, Law and Economics.

Sumário: 1. Introdução: o problema do custo moral. 2. Como identificar custos morais. 3. Como gerir os custos morais. 4. Conclusões: o futuro do diálogo interdisciplinar.

\section{Introdução: o problema do custo moral}

Por que distinguir entre a abordagem da Análise Econômica do Direito (AED) e a do Direito e Economia (D\&E)? Ao atribuir um papel de destaque a essa distinção, Guido Calabresi toca em um ponto fundamental do diálogo interdisciplinar ${ }^{3}$.Quais conceitos usar, o que fazer com eles e quais problemas abordar são algumas das principais escolhas que podem orientar e moldar a interação entre as disciplinas. Na perspectiva de Calabresi, D\&E é o modo de interação a ser privilegiado. Sua principal vantagem é obrigar ambas as disciplinas a confrontarem seus respectivos métodos de análise para obter reconstruções mais precisas da realidade observada. Muitos outros modos de interação são possíveis, com diferenças notáveis do ponto de vista da profundidade das transformações, do grau de integração e

3 CALABRESI, 2016, tr. it 2018, p. 2. 
colaboração entre pesquisadores ${ }^{4}$.Algumas dessas interações abriram caminhos para novos campos de investigação interdisciplinar ou deram origem a disciplinas separadas das matrizes originais. Para alguns temas, o AED criticado por Calabresi poderia ser perfeitamente legítimo como uma estratégia interna (não interdisciplinar) capaz de refinar modelos econômicos ${ }^{5}$.

O livro de Calabresi explora os problemas metodológicos de uma das formas mais avançadas de interdisciplinaridade: a abordagem de D\&E exige que pesquisadores de ambas as disciplinas colaborem tanto na definição das ferramentas de análise quanto nos problemas a serem analisados. O resultado esperado desta colaboração é uma transformação profunda em ambos os lados. Para indicar como essa transformação deve ocorrer, Calabresi escolhe temas que estão localizados nas áreas de interseção entre os sistemas tradicionais de alocação de recursos. Em outras palavras, essas são as áreas nas quais as disputas sobre objetivos e ferramentas para alcançá-los se tornam mais visíveis: bens meritórios (merit goods) que não podem ser administrados com estruturas de mercado puro ou autoritárias, preferências altruísticas que justificam o uso de organizações sem fins lucrativos, valores tidos como relevantes para a organização de uma sociedade, mas não considerados adequadamente

4 Cf. CSERNE, 2020, p. 9-30 (oito formas diferentes de interação entre direito e economia).

5 Por exemplo, considerar que as características do contexto institucional contribuem, por vezes de forma decisiva, para o desenvolvimento econômico, permite não limitar a análise a fatores como a dotação de recursos naturais ou a disponibilidade de capital físico e humano. As vantagens das teorias econômicas institucionalistas sobre teorias alternativas devem ser reconhecidas mesmo que a noção de 'instituição' seja controversa no debate econômico (VOIGT, 2019, p. 12-19) e nem sempre coincide com as reconstruções que os juristas consideram relevantes (Cf. TAMANAHA, 2015; DEAKIN et al., 2017). 
nos processos de tomada de decisão coletiva. Em todas as áreas mencionadas, as dificuldades metodológicas do diálogo interdisciplinar são de dois tipos:

a) Como analisar situações em que os benefícios e custos individuais são diretamente influenciados pelos benefícios e custos de outros indivíduos, grupos ou da sociedade em geral. Onde esta interdependência da utilidade pessoal se manifesta, Calabresi propõe recorrer à noção de custo moral externo para torná-la visível.

b) Como determinar quais estruturas jurídicas são capazes de gerir os custos morais externos. Calabresi há muito tempo tornou explícita sua preferência por estruturas de mercado modificadas ou estruturas autoritárias modificadas ${ }^{6}$.

Em muitas áreas, a relevância dessas duas dificuldades metodológicas é facilmente identificada. Se os objetivos de desenvolvimento sustentável forem perseguidos por meio da transformação dos mercados e da regulamentação, um impacto nas interdependências entre indivíduos e grupos é altamente provável. Se é pedido aos cidadãos que contribuam com a sustentabilidade com suas escolhas de consumo, devese encontrar uma forma de influenciar o comportamento das massas sem reduzir os espaços de liberdade necessários nas sociedades democráticas. Se é pedido às empresas globais que sejam socialmente responsáveis ${ }^{7}$, devem ser encontradas formas de influenciar os modelos organizacionais para torná-los compatíveis com os valores escolhidos. Para que

6 Cf. CALABRESI e BOBBITT, 2006, p. 53ss. (modified political devices) e p. 83ss. (modified markets).

7 Ou, na terminologia de CALABRESI, 2016, tr. it 2018, p. 105ss., para prestar atenção às preferências 'altruístas'. 
se desfrutem dos benefícios das tecnologias digitais, novas formas de discriminação e desigualdade devem ser evitadas. Se a integração dos mercados a nível regional ou global deve ser acompanhada pelo desenvolvimento de novas noções de justiça social, é necessário identificar as estruturas jurídicas capazes de garantir a coexistência dos dois objetivos.

Para cada um dos problemas mencionados, o referencial teórico proposto por Calabresi certamente não é o único instrumento de análise. É possível, no entanto, argumentar que, quando esses problemas são enfrentados com uma abordagem interdisciplinar, é sempre necessário definir os valores de referência e identificar as estruturas jurídicas compatíveis com a sua implementação. Ou seja, as duas dificuldades metodológicas mencionadas são sempre identificadas para qualquer proposta teórica que queira considerar conjuntamente um conjunto de valores e os espaços a serem reservados para o mercado e a regulação.

As seções seguintes exploram a possibilidade de usar a comparação jurídica para identificar os custos morais e as estruturas jurídicas que devem regê-los. Duas considerações justificam o uso de uma abordagem comparativa nesse âmbito. Em primeiro lugar, os custos morais são observáveis apenas de modo indireto, isto é, com base nas escolhas coletivas que colocam à disposição determinados bens ou criam determinados custos. De acordo com Calabresi:

As estruturas jurídicas existentes sugerem uma série de decisões sobre quais ativos e passivos podem garantir àquela sociedade em particular a maior redução dos custos da desigualdade em relação aos benefícios dos incentivos no caso de serem retirados do mercado em menor ou maior grau. Ao mesmo tempo, porém, eles nos dizem muito mais sobre quais incentivos essa sociedade específica acredita que precisa em relação aos custos morais ligado à desigualdade. Nesse sentido, as diversas sociedades apresentam diferenças sejam entre si sejam em diferentes circunstâncias. ${ }^{8}$

8 CALABRESI, 2018, p. 88s. CALABRESI e BOBBITT, 1978, p. 178 ('how 
Essa indicação pode ser utilizada para explorar a relação bilateral entre custos morais e estruturas legais. Se as regras adotadas em dois ordenamentos jurídicos divergem, é possível que a diferença se deva à maior ou menor importância dos custos morais? Dar uma resposta afirmativa a essa questão significa avaliar as escolhas institucionais de cada sistema sob um novo ponto de vista. Em outras palavras, trata-se de estabelecer se os custos morais desempenham um papel decisivo e quais as consequências que eles produzem na alternativa entre mercado e regulação.

Em segundo lugar, as estruturas jurídicas modificadas de Calabresi podem estar disponíveis em maior ou menor grau em cada sistema. Além disso, é possível que cada sistema deva adotar uma estrutura legal modificada com características particulares. A comparação deve servir não apenas para identificar os fatores que influenciam a escolha entre a estrutura de mercado ou autoritária, mas também as características de cada estrutura jurídica em um sistema específico.

A comparação que usa os custos morais como ponto de referência assume tons prescritivos. Considerar a desigualdade relevante para a escolha das estruturas jurídicas significa aderir a uma das teorias morais que considera injustificada uma distribuição específica da riqueza. ${ }^{9}$ Essa premissa metodológica também é necessária quando um sistema jurídico reconhece explicitamente o princípio da igualdade ou declara que deseja perseguir objetivos de justiça social. A análise dos custos morais requer a avaliação de onde e

different conceptions of equality interplay with different institutional and legal traditions to make some approaches to tragic choices plausible for a society while excluding others').

9 Cf. SCANLON, 2018 (discussão de seis razões diferentes para se opor a diferentes formas de desigualdade). 
como se daria a atuação para tais objetivos. É possível, entretanto, evitar uma abordagem exclusivamente prescritiva e reconhecer a tarefa de coletar as informações necessárias para identificar os custos sociais da comparação. O principal objetivo de Calabresi é deslocar a análise econômica para os aspectos mais complexos da realidade jurídica. Observar essa complexidade de fora pode ajudar a distinguir as escolhas institucionais mais diretamente influenciadas pelos custos morais. Em uma inspeção mais próxima, essa é a mesma perspectiva cultivada pelos estudos de Comparative Law and Economics. Embora este campo de investigação tenha sido por vezes vítima da abordagem unilateral criticada por Calabresi, permanecem intactas as razões que aconselham a não limitar a análise econômica a um único ordenamento jurídico ${ }^{10}$. Estudos sobre a psicologia dos processos cognitivos e de tomada de decisão já permitiram estender as formas de interação entre comparação e análise econômica ${ }^{11}$. É plausível levantar a hipótese de que uma extensão análoga pode derivar da análise dos custos morais.

A segunda parte deste texto concentra a atenção na primeira dificuldade metodológica. Mais especificamente, define-se a noção de custo moral, descreve-se o debate suscitado por essa noção e propõe-se a utilização de uma análise em três fases para estabelecer a relevância dos custos morais em cada ordenamento jurídico. A terceira parte discute os fatores institucionais que podem orientar a escolha das estruturas jurídicas às quais se confia a tarefa de gerir os custos morais. A quarta parte sintetiza a análise e oferece algumas reflexões sobre as perspectivas desta área de pesquisa.

10 CATERINA, 2012; RAMELLO, 2016; FAUST, 2019.

11 Cf. DE CONINCK, 2011; ENGEL e SCHMELZER, 2017/16; MOSER, 2018; RÜHL, 2018; LINARELLI, 2019. 


\section{Como identificar custos morais}

Os custos morais representam a reação negativa associada à escolha de disponibilizar determinados bens por meio do mercado ou por meio de estruturas autoritárias. Para Calabresi, a reação negativa tem duas causas: a recusa em fixar um preço para determinados bens e a relutância em aceitar que determinados bens estejam disponíveis em maior quantidade para quem pode pagar mais. Os mesmos bens podem determinar, ambos, os custos morais: por exemplo, a venda de órgãos humanos provoca ambas as reações negativas. $\mathrm{O}$ mesmo pode ser dito sobre o acesso aos recursos hídricos: alguns podem rejeitar a ideia de que a água é uma mercadoria a ser precificada, mas também acreditam que o acesso não deve depender de renda.

Esta definiçãa de custos morais levanta duas questões, ambas discutidas nesta seção:

1. Quais são os custos morais a considerar? Todos ou apenas alguns?

2. Como interpretar a relação bilateral entre custos morais e estruturas jurídicas?

Vamos considerar a primeira questão. Calabresi acredita que a noção de bem meritório ajuda a reconhecer a importância dos custos morais. Essa categoria de bens foi introduzida na década de 50 pelo economista de origem alemã Richard A. Musgrave (1910-2007). É importante lembrar as características das diferentes categorias de bens propostas pela teoria econômica (Tabela 1). Para bens privados, públicos e semipúblicos (em italiano "beni di club"), a classificação é baseada em duas características que afetam sua oferta. Bens meritórios podem compartilhar as características de não ri- 
validade e não exclusividade. No entanto, ao contrário das outras categorias, a classificação considera o lado da demanda. O consumo de bens meritórios é insuficiente porque não leva em consideração as externalidades positivas difusas que pode gerar. Se trata de bens para os quais se torna essencial reconhecer a interdependência das preferências individuais. Os benefícios dos bens meritórios não estão relacionados ao seu consumo direto, mas à influência que o seu consumo por terceiros exerce nas relações sociais. ${ }^{12}$

\section{Tabela 1. Classificação de bens na teoria econômica.}

\begin{tabular}{|c|c|c|}
\hline Categorias de bens & Característica & Exemplo \\
\hline Bens privados & Rivais e excludentes & Edifícios, veículos \\
\hline Bens públicos & $\begin{array}{c}\text { Não rivais e não } \\
\text { excludentes }\end{array}$ & Ideias \\
\hline $\begin{array}{c}\text { Bens semipúblicos } \\
\text { Gestões Coletivas }\end{array}$ & $\begin{array}{c}\text { Parcialmente rivais e } \\
\text { excludentes }\end{array}$ & $\begin{array}{c}\text { Internet } \\
\text { Recursos naturais }\end{array}$ \\
\hline Bens meritórios & $\begin{array}{c}\text { Não rivais e não } \\
\text { excludentes, } \\
\text { consumo e oferta } \\
\text { insuficientes }\end{array}$ & $\begin{array}{c}\text { Educação, sistema } \\
\text { de saúde, proteção } \\
\text { ambiental }\end{array}$ \\
\hline
\end{tabular}

A definição de bens meritórios usada por Calabresi não coincide com a definição de Musgrave. Este último inseriu a noção dentro de uma abordagem da economia do

12 FRISCHMANN, 2012, p. 49. A categoria de bens relacionais, introduzida na década de 1980 e explorada pela literatura econômica e sociológica (Cf. FIORITO e VATIERO, 2013, p. 87; DONATI, 2019), enfatiza a interação positiva e negativa entre o consumo individual e o consumo dos outros. É, portanto, uma noção que poderia justificar formas de intervenção análoga às discutidas por Calabresi. 
bem-estar que justificava a intervenção do Estado apenas no caso de falha do mercado. Embora Musgrave tenha identificado várias razões para justificar a distribuição de bens meritórios, suas propostas exigiam considerar explicitamente as preferências sociais incompatíveis com as preferências individuais. ${ }^{13} \mathrm{Na}$ ausência de uma teoria moral que permitisse a seleção de preferências sociais relevantes, a categoria de bens meritórios poderia ser considerada consistente com as premissas teóricas da economia do bem-estar apenas na condição de ser mantida dentro de limites circunscritos. Calabresi faz a operação inversa, ou seja, se propõe a introduzir no debate uma noção de bem meritório que não pressupõe uma falha de mercado e não requer, em todos os casos, a intervenção do Estado. Não é surpreendente que uma das objeções levantadas a Calabresi seja a amplitude excessiva de sua noção de um bem meritório. Se sua distribuição pelo Estado não se limita aos casos em que não produz distorções no mercado, a intervenção pública pode favorecer grupos de interesse ou gerar corrupção. ${ }^{14}$

Essa objeção não distingue, como se propõe neste artigo, entre a identificação dos custos morais e os instrumentos para governá-los. Também não considera a variedade de estruturas jurídicas modificadas discutidas por Calabresi. Em termos mais gerais, a análise dos custos morais não pode ser feita a partir de uma noção restritiva de um bem meritório. A análise dos custos morais serve para reconhecer situações em que as preferências individuais são interdependentes, ou seja, precisamente as situações em que o mercado e a regulação governam com enorme dificuldade. Obviamente, é necessário identificar os limites da categoria dos custos morais. Alguns limites não deveriam ser con-

13 DESMARAIS-TREMBLAY, 2019.

14 LEVMORE, 2017. 
troversos: preferências racistas ou discriminatórias, ou em geral vetadas por princípios de categoria constitucional e medidas legislativas específicas, não poderiam ser levadas em consideração. ${ }^{15}$ Mais problemáticas são as preferências que envolvem escolhas individuais éticas ou religiosas. Por exemplo, devemos considerar os custos morais de quem não tolera pornografia, casamento entre pessoas do mesmo sexo, consumo de álcool, uso do véu exigido pela religião islâmica, ou trabalho dominical? De acordo com os princípios tradicionais do pensamento liberal, devemos responder que os custos morais só podem ser levados em conta se um determinado comportamento causar danos a outros, mas não se for fruto de escolhas puramente privadas. ${ }^{16}$ No entanto, esse é um limite que corre o risco de restringir excessivamente a noção de custo moral proposta por Calabresi. Se os bens meritórios são necessários para combater formas de desigualdade, vincular sua distribuição a casos em que o dano possa ser comprovado significa renunciar a consideração de um número significativo de interdependências. ${ }^{17}$

De que modo, então, definir os custos morais que consideramos relevantes? Para Calabresi, a resposta pode vir da observação da realidade jurídica. Quando uma estrutura

15 CALABRESI, 2016, tr. it 2018, p. 166. No mesmo sentido POSNER e SUNSTEIN, 2017.

16 A referência é ao "harm principle" de John Stuart Mill. Cf. POSNER e SUNSTEIN, 2017, p. 1817s. e 1835s., bem como a revisão por SUNSTEIN, 2016, p. 53s. TUZET, 2019 observa que algumas interpretações da obra de Mill poderiam justificar o papel que Calabresi atribui aos custos morais, mas conclui que a interferência na liberdade individual é inevitável. Assim como RAMPA, 2016, p. 305, 315-317, pela observação de que a interferência na autonomia individual poderia ser justificada nos casos de interdependência da utilidade pessoal.

17 Como observa CALABRESI, 2017, afirmar que alguém possa se sentir indignado com o comportamento dos outros significa reiterar que não há avaliação neutra das preferências individuais: uma escolha coletiva deve ser feita quanto às preferências a que se dá prioridade. 
legal modificada é usada para distribuir um bem meritório, é possível supor que a relevância dos custos morais deve ser levada em consideração. Aqui encontramos a segunda dificuldade mencionada acima, a saber, a relação bilateral entre custos morais e estruturas jurídicas. Trata-se de uma relação bilateral porque é plausível hipotetizar influências recíprocas: os custos morais são relevantes porque se refletem nas estruturas jurídicas ou as estruturas jurídicas influenciam as preferências individuais em relação aos custos morais? Essa reciprocidade pode alimentar o ceticismo sobre a utilidade dos custos morais como instrumento de análise. Assumir que as estruturas jurídicas levam em consideração os custos morais expõe a objeção para justificar qualquer regime jurídico existente. De que modo é possível levar em conta os custos morais das desigualdades se a realidade jurídica observável já reconhece, por meio de estruturas modificadas, a relevância desses custos? ${ }^{18}$ Alternativamente, é possível dizer que as estruturas jurídicas moldam as preferências individuais e a percepção dos custos morais. Nesse caso, os custos morais não poderiam ser considerados um fator externo capaz de nortear a reforma dos regimes jurídicos existentes.

Uma perspectiva comparativa pode ajudar a identificar os custos morais e avaliar sua relação com as estruturas jurídicas existentes. A análise deve ser dividida em três fases (Figura 1).

18 Sobre o risco de que Law and Economics de Calabresi seja muito conservador, ver MALCAI, 2017. TUZET, 2019, p. 5 observa que avaliar uma proposta de reforma (por exemplo, introdução da poligamia) com base nos custos morais anteriores e subsequentes apresenta problemas significativos de mensuração. O risco é que a incerteza sobre a magnitude desses custos paralise qualquer proposta de reforma. Sobre a circularidade dos custos morais (consequência e ao mesmo tempo causa dos bens meritórios) ver também LANNEAU, 2019. 


\section{Figura 1. Como determinar a relevância dos custos morais.}

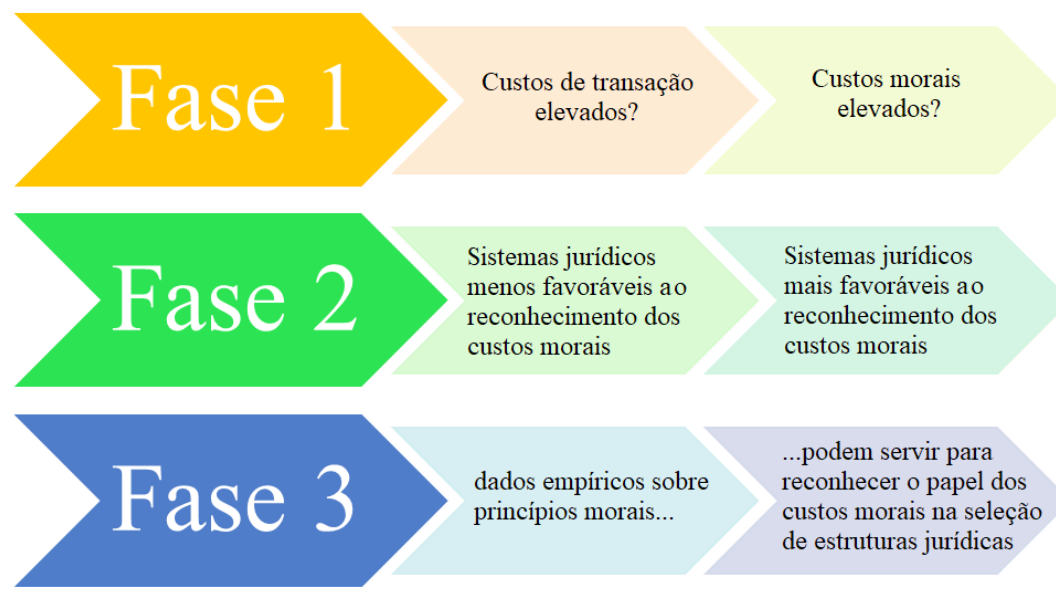

Em primeiro lugar, deve-se reconhecer que a noção de custo moral representa a ferramenta para vincular teoria econômica e reflexão jurídica. Em outras palavras, trata-se de colocar no mesmo nível os aspectos tradicionalmente analisados pela teoria econômica e os aspectos (limites do mercado e da regulação) que a noção de custo moral ajuda a tornar visíveis. Essa estratégia de pesquisa é sugerida por Calabresi ao utilizar as obras de Ronald Coase: se a noção de custo de transação serviu para entender o funcionamento dos mercados e das empresas, a noção de custo moral acrescenta uma explicação alternativa das estruturas jurídicas. ${ }^{19}$ A justaposição entre custos de transação e custos morais ajuda a superar as críticas daqueles que acreditam que estes últimos não podem representar um ponto de referência para a reforma das estruturas jurídicas existentes. Assim como a

19 Cf. CALABRESI, 2016, tr. it 2018, p. 173s.. 
escolha de um regime jurídico pode ser baseada na extensão e natureza dos custos de transação, o mesmo tipo de análise pode ser realizada com referência aos custos morais.

De forma mais aprofundada, os exemplos propostos por Calabresi sugerem uma relação ainda mais próxima entre custos de transação e custos morais. As escolhas coletivas destinadas a reduzir o último podem aumentar o primeiro. Isso significa que os custos de transação e os custos morais costumam se mover em direções opostas. Por exemplo, recorremos organizações sem fins lucrativos para satisfazer preferências altruístas, embora possam ser menos eficientes do que organizações com fins lucrativos. Analogamente, podemos preferir as regras de responsabilidade extracontratual, mesmo quando são mais caras e menos eficientes do que outros sistemas de alocação. ${ }^{20}$ Nesta primeira fase da análise, o objetivo principal é avaliar a relevância dos custos de transação e custos morais em uma área específica, bem como sua interação.

Em segundo lugar, utilizamos a comparação para determinar quais custos morais são relevantes em um determinado contexto legal. Onde os custos morais derivam de desigualdades, é possível concentrar a atenção nas estruturas jurídicas que ajudam a mitigá-los ou agravá-los. Alguns exemplos:

1. Em alguns sistemas jurídicos, os direitos socioeconômicos, associados a condições de vida dignas, são reconhecidos de forma mais limitada do que os direitos civis e políticos. ${ }^{21}$ Por sua tradição constitucional, sua estrutura federativa e seu papel nas relações internacionais, os Estados Unidos é um dos países que concede menos espaço para o reconheci-

20 CALABRESI, 2016, tr. it 2018, p. 107s., 145s..

21 FREDMAN, 2018, p. 59ss.. 
mento de obrigações positivas de proteção. ${ }^{22}$ Outros sistemas jurídicos têm conseguido ter contextos institucionais mais favoráveis ao reconhecimento dos direitos socioeconômicos. Explorar este ponto de partida do ponto de vista dos custos morais permite avaliar se as estruturas jurídicas existentes permitem balancear incentivos de mercado e níveis de desigualdade. Em sistemas jurídicos que deixam pouco espaço para os direitos socioeconômicos, a análise dos custos morais serve para evidenciar o impacto das estruturas jurídicas existentes sobre as desigualdades, sugere como reformá-las, e permite o reconhecimento de caminhos alternativos para reduzir as desigualdades. Um famoso exemplo de caminho alternativo é a inclusão do direito aos benefícios sociais na proteção constitucional concedida à propriedade. ${ }^{23}$ Nos sistemas jurídicos mais propensos a reconhecer os direitos socioeconômicos, a análise dos custos morais não é de forma alguma inútil. Os efeitos desse reconhecimento na melhoria das condições de vida são geralmente modestos. ${ }^{24}$ Mesmo nesses sistemas, portanto, os custos morais podem servir de parâmetro para avaliar a eficácia das estruturas jurídicas existentes.

2. A forma como é decidido reconhecer a prioridade de algumas categorias de credores altera a distribuição da riqueza. A posição vantajosa concedida a alguns grupos pode promover desigualdades. ${ }^{25}$

22 MILLER, 2016, p. 577-593.

23 REICH, 1964, tr. it. 2014; REICH, 1990; RESNIK, 2020, p. 707.

24 Cf. HIRSCHL e ROSEVEAR, 2011, p. 207-228.

25 Cf. os exemplos, principalmente relacionados à regulação dos mercados financeiros, discutidos por PISTOR, 2019, p. 226s. sobre as externalidades 
Um dos fatores que parece contribuir para o sucesso dessas estratégias é a possibilidade de tornar invisível seu impacto negativo na sociedade. $\mathrm{Na}$ pior das hipóteses, os riscos gerados pelas tentativas de escapar da regulamentação causam crises sistêmicas. A análise dos custos morais deve ajudar a reconhecer externalidades negativas e fornecer suporte para uma regulamentação mais rigorosa dos riscos sistêmicos. ${ }^{26}$

3. Qualquer intervenção regulatória requer a seleção do instrumento, ou dos instrumentos, mais adequados para atingir os objetivos desejados. Normalmente, os critérios de seleção consideram a eficiência dos incentivos e os custos de implementação. Observam-se esses dois fatores para decidir se utiliza ferramentas prescritivas (por exemplo, uma tecnologia específica para reduzir a poluição) ou de mercado (por exemplo, o comércio de licenças de emissão), regras decisórias ou obrigatórias, empresas públicas ou privadas, regulamentação pública ou privada. Dentro de cada categoria de instrumentos, outras escolhas são necessárias para calibrar seus efeitos. ${ }^{27}$ Sabemos, porém, que cada

negativas das estratégias de arbitragem regulatória. A reconstrução de Pistor é mais convincente se referente a regimes jurídicos específicos em sistemas jurídicos específicos, menos convincente se pretende descrever a evolução de todos os sistemas jurídicos nos últimos dois séculos. Os regimes legais introduzidos para apoiar os processos de industrialização e o comércio global têm contribuído para a distribuição assimétrica da riqueza também em termos de impacto negativo no clima e nos ciclos naturais: cf. a discussão em VIÑUALES, 2018, p. 32ss.

26 Cf. PISTOR, 2019, p. 225 (“Whoever claims that individual private gains will translate into social welfare improvement should bear the burden of proof for showing the mechanisms by which this feat will be accomplished").

27 Cf., para análise de diferentes perspectivas disciplinares e propostas de 
uma dessas escolhas produz uma distribuição de custos e benefícios entre as diferentes categorias de sujeitos interessados na regulação. Algumas intervenções regulatórias podem ter efeitos regressivos, ou seja, transferir uma parte significativa dos custos para categorias de baixa renda ou aquelas em risco de exclusão social. Além disso, é plausível supor que subsídios cruzados estão presentes em muitos mercados regulamentados. ${ }^{28} \mathrm{~A}$ análise dos custos morais poderia ser usada para avaliar se e como eles influenciam a escolha de ferramentas regulatórias em diferentes sistemas jurídicos. Ou seja, é possível que um instrumento de regulação, ou uma versão específica dele, seja ou não selecionado em função da relevância que os custos morais assumem. Onde esses custos são altos, uma ferramenta eficiente e barata pode se tornar inutilizável. Por outro lado, as ferramentas de intervenção que seriam excluídas porque são incapazes de fornecer incentivos aos agentes racionais poderiam ser levadas em consideração quando a adesão aos princípios morais garante a cooperação. ${ }^{29}$

classificação, cf. SALAMON, 2002; WIENER e RICHMAN, 2010, p. 363-398; WÜRZEL; et al., 2013; DERNBACH, 2018; HOWLETT, 2019; RICHARDS e VAN ZEBEN, 2020.

28 Existe um subsídio cruzado quando dois consumidores do mesmo bem pagam preços diferentes ou pagam o mesmo preço, mas impõem custos diferentes a quem o oferece. Cf., para uma discussão sobre subsídios cruzados em diferentes áreas, ROSSI, 2017; BROOKS et al., 2018; BENSHAHAR e PORAT, 2019.

29 Cf. as observações de SACCONI, 2019, p. 305, 333-335, sobre a possibilidade de que os princípios morais mudem o modo como os agentes concebem as alternativas (de cooperação ou deserção) disponíveis para eles. 
Um levantamento comparativo deve permitir avaliar o equilíbrio entre custos de transação e custos morais em cada sistema jurídico. Em cada uma das áreas exploradas, deve ser possível reconstruir os fatores institucionais que levam a uma preferência por certas formas de destinação de bens meritórios. Além disso, deve-se reconhecer que uma pesquisa comparativa nem sempre será capaz de fornecer informações que excluam explicações alternativas para os custos morais ${ }^{30}$ Uma terceira fase da análise deve servir para verificar se os dados empíricos coletados de outras disciplinas fornecem mais indicações para estabelecer a relevância dos custos morais.

Deve ficar claro que os dados empíricos não devem ser usados para demonstrar que, em uma determinada comunidade, as estruturas jurídicas devem levar em conta os custos morais. É provável que, em qualquer comunidade, as preferências pela distribuição de bens meritórios tende a ser heterogênea. Calabresi reconhece esse problema quando constata a variedade de preferências em relação aos níveis de igualdade. As estruturas legais modificadas devem servir para respeitar o pluralismo das sociedades democráticas contemporâneas. ${ }^{31}$ Os dados empíricos poderiam ser usados para confirmar a relevância das interdependências

30 Cf. LEESON, 2019 pela observação de que a distribuição de bens meritórios e o altruísmo poderiam ser determinados pelas estratégias dos grupos de interesse. No entanto, não há fornecimento de dados empíricos para apoiar esta explicação alternativa.

31 Também é possível argumentar que a decisão de considerar os custos morais deve ser feita com base em escolhas éticas, não necessariamente alinhadas com os valores expressos por uma parte significativa da população: cf. ADLER, 2019, p. 204ss., para a distinção entre ética social e ética crítica. No mesmo sentido, cf. CANEY, 2018, que acrescenta, no entanto, que a pesquisa empírica pode servir para avaliar as implicações dos princípios de justiça, o grau de aceitação, bem como sua relevância em cada contexto. 
entre utilidades pessoais e reconhecer as áreas nas quais o impacto negativo do não reconhecimento dos custos morais é mais provável. Nessa perspectiva, duas áreas de pesquisa oferecem insights valiosos.

Em primeiro lugar, uma ampla literatura coletou dados sobre a dinâmica das desigualdades dentro de cada país, de uma área geográfica ou a nível global. Em uma perspectiva de longo prazo, a mudança dos níveis de desigualdade está associada a mudanças nos regimes políticos e institucionais. Além disso, os níveis de desigualdade são influenciados pelas ideias prevalecentes em cada sociedade sobre as relações entre os diferentes grupos sociais e a legitimidade da distribuição atual da riqueza. ${ }^{32} \mathrm{~A}$ análise dos custos morais traz para este debate uma maior consciência da relação que liga as estruturas jurídicas modificadas e os níveis de desigualdade. Em outras palavras, é possível avaliar se as diferenças nas estruturas jurídicas com tarefas de produção e distribuição de bens meritórios são influenciadas pelas trajetórias de longo prazo que contribuem para definir o nível de desigualdade. Para se limitar ao exemplo italiano, as propostas do Forum Disuguaglianze Diversità para promover a justiça social exigem mudanças nos mecanismos de distribuição de muitos bens meritórios, da educação ao meio ambiente. ${ }^{33}$ Aplicar a análise dos custos morais a essas propostas significa perguntar qual estrutura jurídica modificada tem mais probabilidade de ser compatível com a percepção das desigualdades na Itália. Além disso, uma pesquisa comparativa deve verificar quais estruturas legais

32 Cf. MILANOVIC, 2016 (análise dos ciclos de desigualdade nos últimos séculos); ALVAREDO et al., 2017 (dados sobre desigualdades econômicas em todos os continentes); ATKINSON, 2019 (mensuração das características da pobreza em sessenta países); PIKETTY, 2020 (análise dos fatores que determinaram os níveis de desigualdade nos últimos dois séculos).

FORUM DISUGUAGLIANZE DIVERSITÀ, 2019. 
modificadas foram usadas em outros países para apoiar medidas semelhantes de redução das desigualdades.

Em segundo lugar, estão disponíveis os dados sobre os princípios morais prevalecentes em cada área geográfica. Estudos realizados no campo da teoria econômica, evolução cultural e psicologia moral permitem estabelecer em que medida a população de um determinado país, ou área geográfica menor, apresenta níveis mais ou menos elevados de interdependência de utilidade pessoal. Além disso, esses estudos exploram os fatores sociais, geográficos, demográficos e culturais que podem afetar a formação dos princípios morais. Por exemplo, um estudo realizado em uma amostra representativa de $90 \%$ da população mundial mostra que o altruísmo, a confiança em estranhos e outras preferências 'sociais' (diretamente relacionadas ao comportamento de outras pessoas) são mais comuns em algumas áreas geográficas, mas acima de tudo eles são distribuídos de forma muito diferente dentro de cada país. Também é possível observar correlações significativas entre preferências sociais e atividades beneficentes e voluntárias. ${ }^{34}$ Outro estudo identifica correlações entre princípios morais (a disponibilidade de aceitar deveres de assistência para com estranhos) e o apoio às intervenções do Estado no campo dos benefícios sociais, sistemas de saúde, proteção ambiental e ajuda aos países em desenvolvimento. No entanto, trata-se de uma correlação com diferenças significativas entre os países ocidentais e entre eles e os países não ocidentais. ${ }^{35}$ De forma ainda mais profunda, a literatura em matéria de evolução

34 FALK et al., 2018.

35 ENKE et al., 2019. Em um estudo referente aos Estados Unidos e a quatro países europeus, ALESINA et al., 2018 encontrou uma correlação entre as preferências relacionadas à intervenção estatal e a disposição para aceitar políticas redistributivas. Aqueles que mostram pouca confiança na intervenção do Estado rejeitam as políticas redistributivas, mesmo quando são informados sobre os níveis reais de desigualdade. 
cultural indica que os princípios morais prevalecentes nos países ocidentais, e em particular nos Estados Unidos, determinaram diferenças significativas em relação aos valores prevalecentes em outras áreas geográficas. Fatores sociais (estrutura das relações familiares) e religiosos teriam contribuído para determinar as atitudes em relação à cooperação que observamos em cada país. ${ }^{36}$

Em alguns casos, essas investigações podem fornecer dados empíricos suficientemente precisos para avaliar o impacto da regulamentação sobre os princípios morais, tanto ex-ante como ex-post. ${ }^{37} \mathrm{Na}$ perspectiva de uma análise comparativa, a busca de dados relativos às preferências interpessoais deve permitir ter em conta a heterogeneidade de valores e avaliar em que situações é plausível reconhecer a relevância dos custos morais. Deve-se notar também que os princípios morais adquirem sentido nos diferentes contextos que requerem a sua aplicação. ${ }^{38}$ Considerar que os aspectos institucionais representam um componente relevante de tais contextos significa lidar com a complexidade da interação entre fatores externos e internos em um sistema jurídico. Pelo menos em primeiro lugar, é plausível levantar a hipótese de uma causalidade bilateral entre regras e princípios morais. Os dados empíricos podem oferecer indicações da direção predominante em uma determinada área. Mas a possibilidade de influências recíprocas abre caminho para estratégias regulatórias que visam fortalecer alguns princípios morais (por exemplo, em favor de sociedades mais justas) e reduzir a

36 Cf. SCHULZ et al., 2019; MUTHUKRISHNA et al. Os mecanismos de evolução cultural, em particular no que se refere à vontade de cooperar, podem estar ligados a mudanças nas preferências relativas a um amplo leque de políticas públicas: cf. CLAESSENS et al., 2020.

37 Cf. FABBRI, 2019.

38 Cf. INBAR, 2018; SCHEIN, 2020. 
relevância de outros (por exemplo, a relutância em cooperar além do próprio círculo familiar). ${ }^{39}$

As três fases em que se pode organizar a análise dos custos morais devem permitir estabelecer a sua relação com os custos de transação, identificar os contextos institucionais mais favoráveis ao seu reconhecimento e utilizar dados empíricos sobre princípios morais para selecionar as áreas nas quais é plausível levantar a hipótese da sua relevância. Uma vez estabelecida a importância dos custos morais, deve ser discutira a alternativa entre a estrutura legal de mercado ou autoritária. O próximo ponto é dedicado a este tema.

\section{Como gerir os custos morais}

É possível traçar uma lista de fatores para orientar a escolha de uma estrutura jurídica modificada? Este ponto resume as indicações feitas por Calabresi e oferece algumas indicações adicionais. Além disso, devem ser consideradas as correlações entre os dois tipos de estruturas jurídicas modificadas.$^{40}$ Isso será discutido na parte final desta seção.

A busca por estruturas jurídicas capazes de sustentar os valores a serem promovidos poderia contemplar os seguintes fatores:

a) A transparência da decisão sobre a extensão e atribuição dos custos morais. Se a quantificação for inaceitável, é necessário usar processos de tomada de decisão que reduzam o impacto negativo sobre aqueles que se opõem a métodos específicos de

39 Cf. as observações de EFFERSON et al., 2020 sobre as estratégias que podem ser utilizadas para modificar os princípios morais de um grupo social com preferências heterogêneas. As estruturas legais modificadas podem ser consideradas um exemplo de tais estratégias.

40 CALABRESI, 2016, tr. it 2018, p. 110, 112. 
distribuição de bens meritórios. A flexibilidade dos critérios de quantificação das compensações extracontratuais é uma das alternativas disponíveis. Outra possibilidade é a oferta da integração de princípios morais ou de consideração distributiva nos processos de tomada de decisão dos reguladores. ${ }^{41}$ Neste segundo caso, seria uma estrutura autoritária modificada que leva em consideração a heterogeneidade dos princípios morais. ${ }^{42}$

b) Atribuição de quantidades variáveis de bens meritórios. É possível que uma decisão centralizada seja necessária, mas é plausível que as estruturas de mercado sejam necessárias quando as preferências individuais precisam ser levadas em consideração. ${ }^{43}$

c) Ao recorrer a uma decisão autoritária, é preferível usar o nível institucional que garanta uma distribuição mais equitativa do poder e reduza os custos morais. ${ }^{44}$

41 Cf. POSNER e SUNSTEIN, 2017, para a inclusão de princípios morais na análise de custo-benefício, mas apenas nos casos em que não haja interferência nas liberdades individuais e sem levar em consideração os aspectos distributivos. ADLER, 2019, p. 30-37, observa que a análise de custo-benefício exclui a possibilidade de comparar utilidades pessoais e efeitos distributivos. Esses limites podem ser superados com a adoção de uma função social de bem-estar, ou seja, um procedimento de tomada de decisão que permite a comparação dos benefícios pessoais e exige a escolha de quais princípios éticos priorizar. Deve-se reconhecer que os reguladores frequentemente adotam critérios para avaliar o impacto da distribuição, mas o problema abordado aqui é se esses critérios representam uma resposta satisfatória aos custos morais. Cf. SCHREFLER, 2016; ROBINSON et al., 2016.

42 Até mesmo estratégias de nudging podem ser consideradas uma ferramenta para distribuição de bens meritórios que torna as escolhas coletivas nesta área menos visíveis e, portanto, mais aceitáveis: cf. WHITE, 2019.

43 CALABRESI, 2016, tr. it 2018, p. 65-73.

44 CALABRESI, 2016, tr. it 2018, p. 62. 
d) Atribuição de bens meritórios com base no fator tempo. Calabresi sugere modificar a distribuição para favorecer certas categorias de destinatários. Exemplos relevantes são as regras de acesso prioritário a determinados bens ou serviços, a transição para diferentes formas de tutela com base na expiração de um prazo, ${ }^{45}$ a possibilidade de conceder um período temporário de experimentação a soluções tecnológicas inovadoras. ${ }^{46}$

e) Quais estruturas jurídicas estão menos expostas ao risco de interferência de grupos de interesse e corrupção? Esses problemas geralmente estão associados à intervenção do Estado. Mas Calabresi observa que grupos de interesse podem obter vantagens injustificadas até mesmo nos mercados. $\mathrm{O}$ nível de corrupção depende do tipo de estrutura jurídica usada. ${ }^{47}$

f) Se o comportamento altruísta pode ser desencorajado (fenômeno de crowding-out) ou encorajado (fenômeno de crowding-in) por incentivos (recompensas e penalidades) monetários ou não monetários. Onde os custos morais são altos, qualquer incentivo pode comprometer as motivações pessoais para adotar comportamentos que produzam benefícios para outros. Portanto, é necessário desenhar sistemas de incentivos que reforcem essas motivações. Esses sistemas podem ser integrados tanto nas estrutu-

45 Cf. BELL e PARCHOMOVSKY, 2003.

46 Cf. CORAPI, 2019, p. 785; CREMONA, 2019, p. 547. Sobre a relação entre tempo e regulação tecnológica cf. RANCHORDÁS e ROZNAI, 2020.

47 CALABRESI, 2016, tr. it 2018, p. 175, em resposta às objeç̃es de LEVMORE, 2017, p. 134-138. 
ras jurídicas de mercado e autoritárias, quanto na regulamentação privada. ${ }^{48}$

Todos os fatores mencionados são relevantes para uma pesquisa comparativa. No entanto, deve-se atentar para outro aspecto: nem todas as estruturas jurídicas modificadas estão disponíveis em todos os sistemas jurídicos. Em termos gerais, a distinção entre estruturas autoritárias e de mercado ecoa na disponibilidade de abordagens de direito público e direito privado. Mas essa dicotomia é tratada de forma diferente em cada sistema jurídico. Em outras palavras, é possível encontrar diferenças significativas tanto do lado dos instrumentos de intervenção quanto dos atores públicos e privados chamados a administrá-los. A forma como as diferenças nessa área influenciam a seleção das estruturas jurídicas pode ser observada de vários pontos de vista. Alguns exemplos:

1. A noção de bem meritório proposta por Musgrave foi influenciada pelas reflexões da teoria econômica alemã sobre finanças estatais. Com essa noção, a intervenção estatal poderia ser justificada muito além dos casos de produção de bens públicos não rivais e não excludentes. Era, portanto, uma visão de Estado que só encontrava acolhimento favorável nos meios intelectuais favoráveis às políticas distributivas. ${ }^{49}$ Relativo à noção de bem meritório, um acolhimento certamente maior foi obtido na distinção proposta por Musgrave entre as funções do Estado destinadas a promover a eficiência e as funções do Estado destinadas à distribuição. Essa distinção reflete

48 Cf., para as implicações do crowding-out e a seleção de incentivos, BOWLES, 2016; UNDERHILL, 2016; UNDERHILL, 2019.

49 DESMARAIS-TREMBLAY, 2017. 
os dois teoremas fundamentais da economia do bem-estar, mas também encontrou expressão na diferenciação entre regimes tributários, responsáveis pela distribuição, e outros regimes jurídicos (de direito público ou privado) exclusivamente destinados a garantir uma alocação eficiente de recursos..$^{50}$ Sobre essa distinção, foi contestado que a possibilidade de se buscar políticas distributivas por meio do sistema tributário pode ser prejudicada por uma variedade de fatores institucionais. Os custos morais podem ser mais ou menos altos para cada forma de intervenção redistributiva. Além disso, cada forma de intervenção pode produzir diferentes resultados distributivos. ${ }^{51}$ Estabelecer qual forma de intervenção produz menos distorções de eficiência e ao mesmo tempo garantir um nível aceitável de desigualdade requer informações que muitas vezes não estão disponíveis, ou respostas diferenciadas por setor. ${ }^{52} \mathrm{Um}$ estudo comparativo permite, por um lado, evitar conclusões errôneas sobre a superioridade de uma das formas de intervenção possíveis, por outro lado, permite recolher informações sobre os fatores que podem tornar

50 Cf. DIMICK, 2019 para uma discussão das posições favoráveis e contrárias à divisão de papéis e a conexão com o trabalho de Musgrave. Cf. também ADLER, 2019., p. 225-233, para uma análise que demonstra a necessidade de considerar conjuntamente medidas fiscais e não fiscais.

51 Cf. FENNELL e MCADAMS, 2016 para a discussão dos 'custos da ação política', ou seja, das barreiras à implementação de políticas de distribuição por meio do sistema tributário. Os custos morais de Calabresi se enquadram na categoria mais ampla de custos de ação política (FENNELL e MCADAMS, 2016, p. 1099-1107).

52 Cf. DIMICK, 2019, p. 572 ('there are no strong reasons why the political action costs of legal rules should be systematically lower than those of taxation'). 
mais ou menos disponível uma estrutura jurídica destinada a reduzir as desigualdades. ${ }^{53}$

2. Em ambos os lados do Atlântico, um debate acalorado gira em torno da questão de saber se o direito da concorrência pode ser usado para reduzir as desigualdades. As concentrações excessivas de poder econômico, frequentemente associadas a uma influência política significativa, aumentam as desigualdades, reduzem a taxa de crescimento e desestabilizam as relações sociais. $O$ direito da concorrência poderia, pelo menos indiretamente e em certos mercados, favorecer arranjos mais equilibrados. ${ }^{54}$ Uma referência explícita a objetivos diferentes da eficiência está presente em metade dos países que possuem regras de concorrência. ${ }^{55}$ O Direito da Concorrência Europeu é visto como muito mais disposto do que o Direito Estadunidense a aceitar as metas de justiça social. ${ }^{56}$ No entanto,

53 Por exemplo, o regime tributário europeu em matéria de IVA permite que os Estados-Membros introduzam isenções relacionadas a bens meritórios, mas a erosão da base tributária poderia reduzir os recursos disponíveis para políticas redistributivas (SCARCELLA, 2019). Uma pesquisa comparativa na UE ajudaria a compreender como os Estados-Membros compensam as distorções produzidas pelo sistema fiscal. Um estudo comparativo entre a UE e os Estados Unidos (ou outro sistema jurídico) serviria para entender em que medida os respectivos sistemas fiscais são utilizáveis para a produção de bens meritórios e quais ferramentas alternativas estão disponíveis.

54 BAKER e SALOP, 2016, p. 7. Este trabalho foi debatido em mesa redonda promovida pela mesma revista, com intervenções de AMATO, p. 145; PARDOLESI, p. 148; NICITA, p. 153; OSTI, p. 159; SABBATINI, p. 161. Cf., além disso, as reflexões de PARDOLESI, 2019, p. 81; LIANOS, 2020.

55 BRADFORD et al., 2020. Para obter informações sobre a possibilidade de perseguir objetivos diferentes da eficiência em vários sistemas jurídicos cf. GERADIN e LIANOS, 2019. 
essas condições iniciais não garantem que o Direito da Concorrência seja eficaz na redução dos custos morais. Para se tornar uma estrutura autoritária modificada, capaz de garantir níveis aceitáveis de igualdade sem eliminar os incentivos de mercado, deve incorporar uma avaliação de custos morais compatível com as características das instituições encarregadas de aplicá-la em cada ordenamento jurídico. Nos Estados Unidos, o principal obstáculo para essa evolução pode ser a aplicação descentralizada da legislação antitruste. ${ }^{57}$ Outros sistemas jurídicos poderiam incorporar mais facilmente a análise de custos morais, desde que os fatores mencionados anteriormente sejam usados para avaliar quais estruturas jurídicas modificadas são mais adequadas. ${ }^{58}$

3. Nos países industrializados, o transporte público tem historicamente assumido um papel mais (na Europa) ou menos (nos Estados Unidos) relevante. ${ }^{59}$ A digitalização dos serviços de mobilidade e a automatização das funções de condução estão abrindo novas oportunidades para a integração dos serviços de transporte e para a introdução de mecanismos de mercado. Novos modelos de multimodalidade permitem estabelecer preços variáveis

57 Cf. MELAMED, 2020.

58 A literatura sociológica aponta que as preferências em favor da concorrência e das políticas distributivas podem estar associadas em diferentes graus em cada país (KOOS e SACHWEH, 2019). Retorna aqui o problema da relação bilateral entre princípios morais e instituições. Contudo, é plausível levantar a hipótese de que o uso do direito da concorrência para combater as desigualdades depende da percepção da legitimidade das políticas de distribuição implementadas com outras ferramentas.

59 KANGER ET AL., 2019; WONG ET AL., 2020. 
de acesso às infraestruturas rodoviárias e de cada meio de transporte. ${ }^{60} \mathrm{~A}$ vantagem desses mecanismos é refletir com mais precisão os custos externos (econômicos, ambientais e humanos) das escolhas individuais de transporte. Ao mesmo tempo, os novos paradigmas de mobilidade integrada, digital e automatizada tornam mais visíveis as diferentes possibilidades disponíveis para cada grupo social. As infra-estruturas de transporte são 'privatizadas', não no sentido de uma alteração do regime de propriedade, mas no sentido de uma restrição ao acesso com base na disponibilidade para pagar. Esta forma de 'privatização' pode ser percebida como a consolidação das desigualdades já presentes nos sistemas tradicionais de transporte. ${ }^{61}$ Obter as vantagens da inovação tecnológica é possível, mas somente se for acompanhada de uma redistribuição adequada em favor dos grupos de baixa renda ou com menos oportunidades de utilização dos novos modelos de mobilidade. É plausível que este tipo de intervenção seja mais facilmente alcançável se as estruturas de mercado ou autoritárias modificadas refletirem as características dominantes de cada sistema de transporte.

4. Que relevância deve ser atribuída aos custos morais nos países em desenvolvimento? A presença de um maior número de pessoas nas camadas de baixa renda indica que qualquer intervenção pode ser considerada inaceitável se não atentar para os perfis de distribuição. A este aspecto acrescenta-se a neces-

60 SOCIETÀ ITALIANA DI POLITICHE DEI TRASPORTI, 2018; MÜLLER e MEYER, 2019; RIGGS, 2019; FINGER e AUDOUIN, 2019.

61 SPARROW e HOWARD, 2020. 
sidade de considerar outros elementos, geralmente presentes nos países em desenvolvimento: papel mais significativo da economia informal, maior pluralismo jurídico, menor disponibilidade de recursos para instituições públicas. Trata-se, portanto, de contextos econômicos, sociais e institucionais que interagem de formas complexas com processos de tomada de decisão e formação de princípios morais. Diferenças marcantes em relação aos princípios prevalecentes nos países ocidentais foram evidenciadas. ${ }^{62}$ A seleção das estruturas jurídicas modificadas deve levar isso em conta. Considere, por exemplo, a desconfiança generalizada no mercado e nos mecanismos competitivos encontrados nos países da África Subsaariana. ${ }^{63}$ Uma possível resposta é a adoção de soluções institucionais que favoreçam processos competitivos inclusivos, ou seja, visando garantir benefícios difuso para grandes camadas populacionais. No entanto, é preciso reconhecer os riscos dos projetos de reforma que se limitam a promover o fortalecimento da lógica do mercado. ${ }^{64} \mathrm{~A}$ análise dos custos morais poderia ajudar a seguir um caminho diferente de mudança institucional, em que o ponto de partida é representado pela interação entre os princípios morais e o contexto institucional, e o ponto de chegada de uma ou mais estruturas jurídicas modificadas.

62 Além das referências nas notas 32-34, cf. GRAHAM et al., 2018, p.211222 (variedade de princípios morais); KREMER et al., 2019, p. 423-428 (correlação entre preferências sociais e desenvolvimento); HENRICH e MUTHUKRISHNA (abordagem evolutiva das diferenças psicológicas).

63 FOX e BAKHOUM, 2019, p. 159ss.

64 Cf. as observações críticas de BERNDT, 2019, p. 242-256, 250 ('we ultimately observe a more direct interventionist logic that aims at bringing individual behavior in line with a market logic'). 
Esses exemplos sugerem a possibilidade de usar uma pesquisa comparativa não apenas para estabelecer a relevância dos custos morais, mas também para explorar a variedade de estruturas jurídicas modificadas e seus efeitos. No entanto, ainda precisam ser consideradas as possíveis interações entre as estruturas autoritárias e de mercado. Calabresi levanta a hipótese de uma forma de complementaridade: uma maior desigualdade poderia ser aceitável se houvessem mais bens meritórios alocados fora dos mecanismos ordinários do mercado. ${ }^{65}$ No entanto, se trata apenas de uma das interações possíveis. Os exemplos discutidos neste artigo sugerem que as estruturas modificadas estão normalmente inseridas em contextos institucionais com uma pluralidade de relações complementares: regimes de direito público e de direito privado, principalmente orientados para promover a eficiência ou a distribuição de recursos, para limitar ou apoiar os mercados. Mesmo com uma variedade de linguagens e ferramentas analíticas, a comparação jurídica atribui atenção especial a essas complementaridades. A identificação dos custos morais poderia, por um lado, fornecer uma explicação melhor do motivo pelo qual um sistema jurídico construiu uma certa complementaridade, por outro lado, fornecer informações sobre a eficácia de uma determinada forma de complementaridade para garantir o reconhecimento adequado dos custos morais.

\section{Conclusões: o futuro do diálogo interdiscipli- nar}

A análise dos custos morais requer ferramentas que permitam reconhecer sua relevância e selecionar as estruturas jurídicas mais adequadas para geri-los. Neste artigo, foi

65 CALABRESI, 2016, tr. it 2018, p. 90. 
proposto o uso de uma perspectiva comparativa para abordar ambas as dificuldades metodológicas. Quanto à avaliação da relevância dos custos morais, um estudo comparativo pode ser organizado em três fases: comparação entre a entidade dos custos de transação e os custos morais, identificação dos fatores que favorecem ou dificultam o reconhecimento dos custos morais, recolha de dados empíricos que confirmam sua relevância. Quanto à seleção das estruturas jurídicas modificadas, uma pesquisa comparativa pode servir para estabelecer quais as soluções institucionais disponíveis e suas complementaridades.

A forma de diálogo interdisciplinar promovida por Calabresi provoca uma revisão profunda da forma de representação dos problemas a enfrentar, da relação entre direito e economia, bem como das relações com outras disciplinas. A discussão realizada nas seções anteriores mostra que a revisão já está em andamento nas ciências sociais, naturais e humanas. Onde questões morais fundamentais estão em jogo, as pesquisas mais interessantes combinam teorias da evolução, antropologia, etnografia, ciência cognitiva, ciência política, estudos históricos e filosofia moral. Na reflexão econômica, multiplicam-se as posições em favor de uma perspectiva teórica mais inclusiva. ${ }^{66} \mathrm{O}$ êxito deste diálogo não está necessariamente na integração de diferentes disciplinas. ${ }^{67}$ É mais plausível levantar a hipótese de que, em um

66 Cf. BANHERJEE e DUFLO, 2019, p. 9 ('Restoring human dignity to its central place, we argue in this book, sets off a profound rethinking of economic priorities and the ways in which societies care for their members, particularly when they are in need'); NAIDU et al, 2020 ('we consider human prosperity broadly, including nonpecuniary sources of well-being, from health to climate change to political rights'); COLLIER, 2018, tr. it. 2020, p. 30 ("precisamos do mercado, mas vinculado a um propósito firmemente ancorado em bases éticas" - em italiano: "abbiamo bisogno del mercato, ma vincolato ad uno scopo che sia saldamente ancorato a basi etiche").

67 Cf. GRÜNE-YANOFF, 2016 (o diálogo interdisciplinar pode transformar 
futuro próximo, os modos de interação se multiplicarão. A análise dos custos morais, realizada com enfoque de D\&E, pode ser uma das formas mais profícuas de sua contribuição pelo lado da complexidade institucional. Além disso, se a relevância dos custos morais for reconhecida muito além de uma categoria limitada de bens meritórios, o debate iniciado por Calabresi poderia levar a uma abordagem de D\&E muito menos influenciada pela economia de bem-estar tradicional e muito mais útil para lidar com as várias formas de desigualdade. Seria esse o verdadeiro objetivo de Calabresi?

\section{REFERÊNCIAS}

ADLER, Matthew D. Measuring social welfare: An introduction. Oxford University Press, USA, 2019.

ALESINA, Alberto; et al. Intergenerational mobility and preferences for redistribution. American Economic Review, v. 108, n. 2, p. 521-54, 2018.

ALVAREDO, Facundo et al. (Ed.). World inequality report 2018. World Inequality Lab, 2017.

AMATO, Giuliano. Non tocca all'antitrust. Mercato Concorrenza Regole, v. 18, n. 1, 2016.

ATKINSON, Anthony B. Measuring poverty around the world. Princeton University Press, 2019.

BAKER, Jonathan B.; SALOP, Steven C. Antitrust, politica della concorrenza e disuguaglianza. Mercato concorrenza regole, v. 18, n. 1, p. 7-34, 2016.

BANERJEE, Abhijit V.; DUFLO, Esther. Good economics

as disciplinas envolvidas, mesmo que cada uma delas desenvolva suas próprias ferramentas de análise separadamente). 
for hard times: Better answers to our biggest problems. Penguin UK, 2019.

BELL, Abraham; PARCHOMOVSKY, Gideon. Pliability Rules, 101 Mich. L. Rev, v. 1, n. 5, 2003.

BEN-SHAHAR, Omri; PORAT, Ariel. Personalizing mandatory rules in contract law. U. Chi. L. Rev., v. 86, p. 255, 2019. BERNDT, Christian. Behavioural economics and development policy. STRAßHEIM, Holger; BECK, Silke. Introduction to the handbook of behavioural change and public policy. Handbook of behavioural change and public policy. Edward Elgar Publishing, 2019.

BOWLES, Samuel. The moral economy: Why good incentives are no substitute for good citizens. Yale University Press, 2016.

BRADFORD, Anu; et al. The Chicago School's Limited Influence on International Antitrust. The University of Chicago Law Review, v. 87, n. 2, p. 297-330, 2020.

BROOKS, John et al. Cross-Subsidies: Government's Hidden Pocketbook. Geo. LJ, v. 106, p. 1229, 2018.

CALABRESI, Guido e BOBBITT, Philip. Tragic Choices, New York e London, 1978. Tradução em italiano: Scelte tragiche, $2^{\circ}$ ed., Milano, 2006.

CALABRESI, Guido. The Future of Law and Economics: Comments and Reflections. Jerusalem Review of Legal Studies, v. 16, n. 1, p. 167-178, 2017.

CALABRESI, Guido.The future of law and economics: Essays in reform and recollection. Yale University Press, 2016. Tradução em italiano: FIMMANÒ, F.; OCCORSIO, V. Il Futuro del Law and Economics. 2018. 
CANEY, Simon. Global Distributive Justice: Seven Theses about Facts and Empirical Research. The Oxford Handbook of International Political Theory, p. 103-17, 2018.

CATERINA, Raffaele. Comparative law and economics. Elgar Encyclopedia of Comparative Law, $2^{\circ}$ ed., Edward Elgar Publishing, 2012.

CLAESSENS, Scott et al. The dual evolutionary foundations of political ideology. Nature Human Behaviour, p. 1-10, 2020.

COLLIER, Paul. The future of capitalism: Facing the new anxieties. Penguin UK, 2018. Tradução em italiano: Il futuro del capitalismo: fronteggiare le nuove ansie, Bari/Roma, 2020 (versão ePub)

CORAPI, E. Regulatory Sandbox nel Fintech?. Diritto del commercio internazionale, 2019.

CREMONA, Brando Maria. RegTech 3.0: verso un Regulatory Sandbox europeo?. Mercato Concorrenza Regole, v. 21, n. 3, p. 547-577, 2019.

CSERNE, Péter. Knowledge claims in Law and Economics: Gaps and bridges between theoretical and practical rationality. Law and Economics as Interdisciplinary Exchange. Routledge, 2019. p. 9-30.

DECONINCK, Julie. Reinvigorating comparative law through behavioral economics? A cautiously optimistic view. Review of Law \& Economics, v. 7, n. 3, p. 711-736, 2011.

DEAKIN, Simon et al. Legal institutionalism: Capitalism and the constitutive role of law. Journal of Comparative Economics, v. 45, n. 1, p. 188-200, 2017.

DERNBACH, John C. The Dozen Types of Legal Tools in the Deep Decarbonizaiton Toolbox. Energy LJ, v. 39, p. 313, 2018. 
DESMARAIS-TREMBLAY, Maxime. A genealogy of the concept of merit wants. The European journal of the history of economic thought, v. 24, n. 3, p. 409-440, 2017.

DESMARAIS-TREMBLAY, Maxime. The normative problem of merit goods in perspective. Forum for Social Economics. V. 48, p. 219, 2019.

DIMICK, Matthew. The Law and Economics of Redistribution. Annual Review of Law and Social Science, v. 15, p. 559-582, 2019.

DONATI, Pierpaolo, Scoprire i beni relazionali: per generare una nuova socialità. Soveria Mannelli, 2019

EFFERSON, Charles; et al. The promise and the peril of using social influence to reverse harmful traditions. Nature human behaviour, v. 4, n. 1, p. 55-68, 2020.

ENGEL, Christoph; SCHMELZER, André. Committing the English and the continental way: An experiment. Preprints of the Max Planck Institute for Research on Collective Goods, Bonn, 2017/16.

ENKE, Benjamin; et al. Moral Universalism and the Structure of Ideology. Cesifo Working Papers n. 7924, 2019.

FABBRI, Marco. Shaping Tastes and Values Through the Law: Law and Economics Meets Cultural Economics. Global Jurist, v. 19, n. 3, 2019.

FALK, Armin et al. Global evidence on economic preferences. The Quarterly Journal of Economics, v. 133, n. 4, p. 1645-1692, 2018.

FAUST, Florian. Comparative law and economic analysis of law. The Oxford handbook of comparative law. $2^{\circ}$ ed., Oxford, 2019. 
FENNELL, Lee Anne; MCADAMS, Richard H. The distributive deficit in law and economics. Minn. L. Rev., v. 100, p. 1051, 2016.

FINGER, Matthias; AUDOUIN, Maxime (Ed.). The Governance of Smart Transportation Systems: Towards New Organizational Structures for the Development of Shared, Automated, Electric and Integrated Mobility. Springer, 2019.

FIORITO, Luca; VATIERO, Massimiliano. A joint reading of positional and relational goods. Economia politica, v. 30, n. 1, p. 87-96, 2013.

FORUM DISUGUAGLIANZE DIVERSITÀ, 15 Proposte per la Giustizia Sociale - Ispirate dal Programma di Azione di Anthony Atkinson, Roma, 2019.

FOX, Eleanor M.; BAKHOUM, Mor. Making markets work for Africa: markets, development, and competition law in Sub-Saharan Africa. Oxford University Press, 2019.

FREDMAN, Sandra. Comparative Human Rights Law. Oxford University Press, 2018.

FRISCHMANN, Brett M. Infrastructure: The social value of shared resources. Oxford University Press, 2012.

GERARD, Damien; LIANOS, Ioannis (Ed.). Reconciling Efficiency and Equity: A Global Challenge for Competition Policy. Cambridge University Press, 2019.

GRAHAM, Jesse et al. Moral foundations theory: on the advatanges of moral pluralism over moral monism. GRAY, Kurt; GRAHAM, Jesse (Ed.). Atlas of moral psychology. The Guilford Press, 2018.

GRÜNE-YANOFF, Till. Interdisciplinary success without integration. European Journal for Philosophy of Science, v. 6, n. 3, p. 343-360, 2016. 
HENRICH, Joseph; MUTHUKRISHNA, Michael. The origins and psychology of human cooperation. A ser publicado no Annual Review of Psychology, 2021.

HIRSCHL, Ran; ROSEVEAR, Evan. Constitutional Law Meets Comparative Politics: Socio-Economic Rights and Political Realities (Chapter 10). CAMPBELL, Tom; Ewing, Keith D.; TOMKINS, Adam. The Legal Protection of Human Rights: Sceptical Essays, Oxford, 2011.

HOWLETT, Michael. Designing public policies: Principles and instruments. Routledge, 2019.

INBAR, Yoel. Applied moral psychology. GRAY, Kurt; GRAHAM, Jesse (Ed.). Atlas of moral psychology. The Guilford Press, 2018.

KANGER, Laur et al. Technological diffusion as a process of societal embedding: Lessons from historical automobile transitions for future electric mobility. Transportation Research Part D: Transport and Environment, v. 71, p. 47-66, 2019.

KOOS, Sebastian; SACHWEH, Patrick. The moral economies of market societies: popular attitudes towards market competition, redistribution and reciprocity in comparative perspective. Socio-Economic Review, v. 17, n. 4, p. 793-821, 2019.

KREMER, Michael et al. Behavioral Development Economics. BERNHEIM, B. Douglas; DELLAVIGNA, Stefano; LAIBSON, David. Handbook of Behavioral EconomicsFoundations and Applications 2. Elsevier, 2019.

LANNEAU, Régis. To What Extent Should We Enrich Law and Economics? On Calabresi and his Future of Law and Economics. Global Jurist, v. 19, n. 3, 2019. 
LEESON, Peter T. Do we need behavioral economics to explain law?. European Journal of Law and Economics, p. 1-14, 2019.

LEVMORE, Saul. Merit Goods, Calabresi-Goods, and RentSeeking: In Honor of Guido Calabresi's The Future of Law and Economics. Jerusalem Review of Legal Studies, v. 16, n. 1, p. 129-141, 2017.

LIANOS, Ioannis. Competition Law as a Form of Social Regulation. The Antitrust Bulletin, v. 65, n. 1, p. 3-86, 2020.

LINARELLI, John. Behavioral Comparative Law: Its Relevance to Global Commercial Law-Making. In: N. Orkun Akseli \& John Linarelli, The Future of Commercial Law: Ways Forward for Change and Reform. Hart 2019 Forthcoming, 2019.

MALCAI, Ofer. The Alternative Futures of Law and Economics: Comments on Guido Calabresi's The Future of Law and Economics. Jerusalem Review of Legal Studies, v. 16, n. 1, p. 83-100, 2017.

MELAMED, A. Douglas. Antitrust Law and Its Critics. A ser publicado em 83 Antitrust L.J., 2020.

MILANOVIC, Branko. Global inequality: A new approach for the age of globalization. Harvard University Press, 2016. MILLER, Jonathan M. The influence of human rights and basic rights in private law in the United States. The American Journal of Comparative Law, v. 15, p. 577-593, 2016.

MOSER, Gustavo., Rethinking Choice of Law in CrossBorder Sales, Den Haag, 2018.

MÜLLER, Beate; MEYER, Gereon. Towards User-Centric Transport in Europe. Springer, Cham, 2018. 
MUTHUKRISHNA, Michael et al. Beyond WEIRD psychology: measuring and mapping scales of cultural and psychological distance. A ser publicado em Psychol. Sc.

NAIDU, Suresh; et al. Economics after Neoliberalism: Introducing the EfIP Project. AEA Papers and Proceedings. 2020. p. 366-71.

NICITA, Antonio. Il rischio di una discrezionalità eccessiva. Mercato Concorrenza Regole, v. 18, n. 1, 2016.

OSTI, Cristoforo. Perché no?. Mercato Concorrenza Regole, v. 18, n. 1, 2016.

PARDOLESI, Roberto. Hipster antitrust e sconvolgimenti tettonici: «back to the future»?. Mercato Concorrenza Regole, v. 21, n. 1, p. 81-94, 2019.

PARDOLESI, Roberto. Non rinunciamo a provarci. Mercato Concorrenza Regole, v. 18, n. 1, 2016.

PIKETTY, Thomas. Capital and ideology. Harvard University Press, 2020.

PISTOR, Katharina. The code of capital: How the law creates wealth and inequality. Princeton University Press, 2019. POSNER, Eric A.; SUNSTEIN, Cass R. Moral commitments in cost-benefit analysis. Va. L. Rev., v. 103, p. 1809, 2017.

RAHMAN, K. Sabeel; THELEN, Kathleen. The rise of the platform business model and the transformation of twenty-first-century capitalism. Politics \& Society, v. 47, n. 2, p. 177-204, 2019.

RAMELLO, Giovanni B. The past, present and future of comparative law and economics. Comparative Law and Economics. Edward Elgar Publishing, 2016.

RAMPA, Lorenzo. Paternalismo, autonomia e diritti sociali: una rilettura in termini di analisi economica. Politica del diritto, v. 47, n. 3, p. 305-336, 2016. 
RANCHORDÁS, Sofia; ROZNAI, Yaniv (Ed.). Time, Law, and Change: An Interdisciplinary Study. Bloomsbury Publishing, 2020.

REICH, Charles A. The new property after 25 years. USFL Rev., v. 24, p. 223, 1990.

REICH, Charles A. The new property. The Yale Law Journal, v. 73, n. 5, p. 733-787, 1964. Tradução em italiano: D'URSO, Francesco. La nuova proprietà, Torino, 2014.

RESNIK, Judith. Constituting Security and Fairness: Reflecting on Charles Reich's Imagination and Impact. The Yale L.J. Forum, 2020.

RICHARDS, Kenneth R.; VAN ZEBEN, Josephine. Policy Instruments in Environmental Law. Elgar Encyclopedia of Environmental Law. Edward Elgar Publishing Limited, 2020. p. 1-13.

RIGGS, William (Ed.). Disruptive transport: Driverless cars, transport innovation and the sustainable city of tomorrow. Routledge, 2019.

ROBINSON, Lisa A.; et al. Attention to distribution in US regulatory analyses. Review of Environmental Economics and Policy, v. 10, n. 2, p. 308-328, 2016.

ROSSI, Jim. Carbon Taxation by Regulation. Minn. L. Rev., v. 102, p. 277, 2017.

RÜHL, Giesela. Behavioural analysis and comparative law: improving the empirical foundation for comparative legal research. Research Methods in Consumer Law. Edward Elgar Publishing, 2018.

SABBATINI, Pierluigi. Anti-inequality? Considerazioni sul tema antitrust e diseguaglianza. Mercato Concorrenza Regole, v. 18, n. 1, 2016. 
SACCONI, Lorenzo. Non tutta la massimizzazione viene per nuocere (e i molti significati di "agente massimizzante"). SACCHI R. e TOFFOLETTO A. Esiste uno "stile giuridico" neoliberale?, Atti dei seminari per Francesco Denozza, Milano, 2019.

SALAMON, Lester M. The tools of government: A guide to the new governance. Oxford University Press, 2002.

SCANLON, Thomas. Why does inequality matter?. Oxford University Press, 2018.

SCARCELLA, Luisa. Fundamental Rights and Merit Goods: The Case of the VAT Exemptions in the Public Interest. Global Jurist, v. 19, n. 3, 2019.

SCHEIN, Chelsea. The importance of context in moral judgments. Perspectives on Psychological Science, v. 15, n. 2, p. 207-215, 2020.

SCHREFLER, Lorna. Social impact assessment. DUNLOP, Claire A.; RADAELLI, Claudio M. Handbook of Regulatory Impact Assessment. Edward Elgar Publishing, 2016.

SCHULZ, Jonathan F. et al. The Church, intensive kinship, and global psychological variation. Science, v. 366, n. 6466, 2019.

SOCIETÀ ITALIANA DI POLITICHE DEI TRASPORTI, Innovazioni tecnologiche e governo della mobilità, Rimini, 2018.

SPARROW, Robert; HOWARD, Mark. Make way for the wealthy? Autonomous vehicles, markets in mobility, and social justice. Mobilities, p. 1-13, 2020.

SUNSTEIN, Cass R. Listen, Economists!. New York Review of Books, v. 93, n. 17, p. 53-54, 2016. 
TAMANAHA, Brian Z. The knowledge and policy limits of New Institutional Economics on development. Journal of Economic Issues, v. 49, n. 1, p. 89-109, 2015.

TUZET, Giovanni. Calabresi and Mill: Bilateralism, Moral Externalities and Value Pluralism. Global Jurist, v. 19, n. 3, 2019.

UNDERHILL, Kristen. Money That Costs Too Much: Regulating Financial Incentives. Ind. LJ, v. 94, p. 1109, 2019.

UNDERHILL, Kristen. When extrinsic incentives displace intrinsic motivation: designing legal carrots and sticks to confront the challenge of motivational crowding-out. Yale J. on Reg., v. 33, p. 213, 2016.

VIÑUALES, Jorge E., The Organisation of the Anthropocene: In Our Hands?, Leiden/Boston, 2018.

VOIGT, Stefan. Institutional economics: An introduction. Cambridge University Press, 2019.

WHITE, Mark D. Nudging Merit Goods: Conceptual, Normative, and Practical Connections. Forum for Social Economics. Routledge, 2019

WIENER, Jonathan B.; RICHMAN, Barak D. Mechanism Choice. FARber Daniel A.; O'CONnEll Anne Joseph. Research Handbook on Public Choice and Public Law, Edward Elgar Publishing, 2010.

WONG, Yale Z. et al. Mobility as a service (MaaS): Charting a future context. Transportation Research Part A: Policy and Practice, v. 131, p. 5-19, 2020.

WURZEL, Rüdiger; et al. Environmental governance in Europe: A comparative analysis of the use of new environmental policy instruments. Edward Elgar Publishing, 2013. 
Recebido em 09/09/2020

Aprovado em 06/10/2020

Giuseppe Bellantuono

E-mail: giuseppe.bellantuono@unitn.it

Marcos Henrique Costa Leroy

E-mail:mhcleroy@hotmail.com 
